\title{
Measurement of atmospheric MTF in a littoral environment
}

\author{
Derek J. Griffith ${ }^{*}$, Francois P.J. le Roux ${ }^{1}$, Piet B.W. Schwering ${ }^{2}$ and Mark Holloway ${ }^{1}$ \\ ${ }^{1}$ CSIR Defence, Peace, Safety and Security, P.O. Box 395, Pretoria 0001, South Africa \\ ${ }^{2}$ TNO Defence, Security and Safety, P.O. Box 96864, NL-2509 JG The Hague, Netherlands
}

\begin{abstract}
Measurement of atmospheric modulation transfer function (MTF) derived from the point spread function is an alternative to the use of scintillometry in characterizing the effects of turbulence as well as optical scattering. This experiment involved measurement of the system point spread function at $630 \mathrm{~nm}$ wavelength, over a $1.8 \mathrm{~km}$ path between the shoreline and Roman Rock lighthouse, which is offshore in False Bay near Simonstown, South Africa. Four telescopes at heights between $4 \mathrm{~m}$ and $14 \mathrm{~m}$ above the sea surface were mounted at the shoreline looking at two point sources mounted at heights of $6 \mathrm{~m}$ and $13 \mathrm{~m}$ above the sea surface on the lighthouse. The telescopes were equipped with digital charge-coupled device (CCD) cameras and the system MTF of the combination was measured. While the point spread function is also sensitive to atmospheric scattering, scintillation is driven largely by turbulence. Measurements were made under a wide variety of environmental conditions and the differences between image quality metrics and scintillation metrics were examined. Local weather parameters, visibility and sea temperature were also monitored during the campaign and the impact of these parameters on image quality was assessed. Divergent trends in scintillation and image quality were encountered.
\end{abstract}

Keywords: Atmospheric MTF, turbulence height profile, littoral surveillance

\section{INTRODUCTION}

This experiment was designed to give insight into the nature of atmospheric turbulence and specifically the atmospheric MTF over short to medium range in the littoral environment. There were two main aspects of interest. The first aspect was that of accumulating data to get an idea of the severity and variability of turbulence and aerosol effects and their relationship to bulk weather parameters. The second element was to get some insight into the typical variation of atmospheric turbulence with height above sea level. These insights can impact the engineering, positioning and performance of optical surveillance and communication systems in littoral applications.

The measurements were performed during a small surface target campaign undertaken at Simonstown, South Africa during June 2007 (Schwering et. al. ${ }^{1}$ ).

\section{EXPERIMENTAL LAYOUT}

The experimental layout is illustrated in Figure 1. Two light sources were mounted at heights of $6.1 \mathrm{~m}$ and $13.2 \mathrm{~m}$ respectively above mean sea level on Roman Rock Lighthouse. Images of both light sources were recorded using each of four telescopes mounted on the Institute for Maritime Technology (IMT) building in Simonstown over a range of $1826 \mathrm{~m}$. The telescopes were mounted at heights of $4 \mathrm{~m}, 6.9 \mathrm{~m}, 10.7 \mathrm{~m}$ and $14.4 \mathrm{~m}$ above sea level. The sightline from IMT to Roman Rock lay on a bearing of $46.4^{\circ}$ east from north. The area is shown from above in Figure 2 and Figure 3.

Sea temperature was measured at two buoys named Phoenix Buoy and Northern marker in False Bay, at the locations shown in Figure 2 and Figure 3.

Weather stations were situated at Roman Rock on the roof of the lighthouse, on the shoreline next to the IMT building, as well as on the roof of the IMT building. A Vaisala FS11 visibility meter was also operated on the roof of the IMT building.

*dgriffith@csir.co.za; phone +27 12 841-3371; fax +27 12 841-3453; www.csir.co.za

Electro-Optical and Infrared Systems: Technology and Applications V, edited by David A. Huckridge, Reinhard R. Ebert Proc. of SPIE Vol. 7113, 711308 · (c) 2008 SPIE · CCC code: 0277-786X/08/\$18 · doi: 10.1117/12.799797 
Over the same period, TNO operated scintillometers and transmissometers over a long path of $9.7 \mathrm{~km}$ between IMT and the Empire building on the shoreline at Muizenberg ${ }^{1}$. This path is also shown on Figure 3.

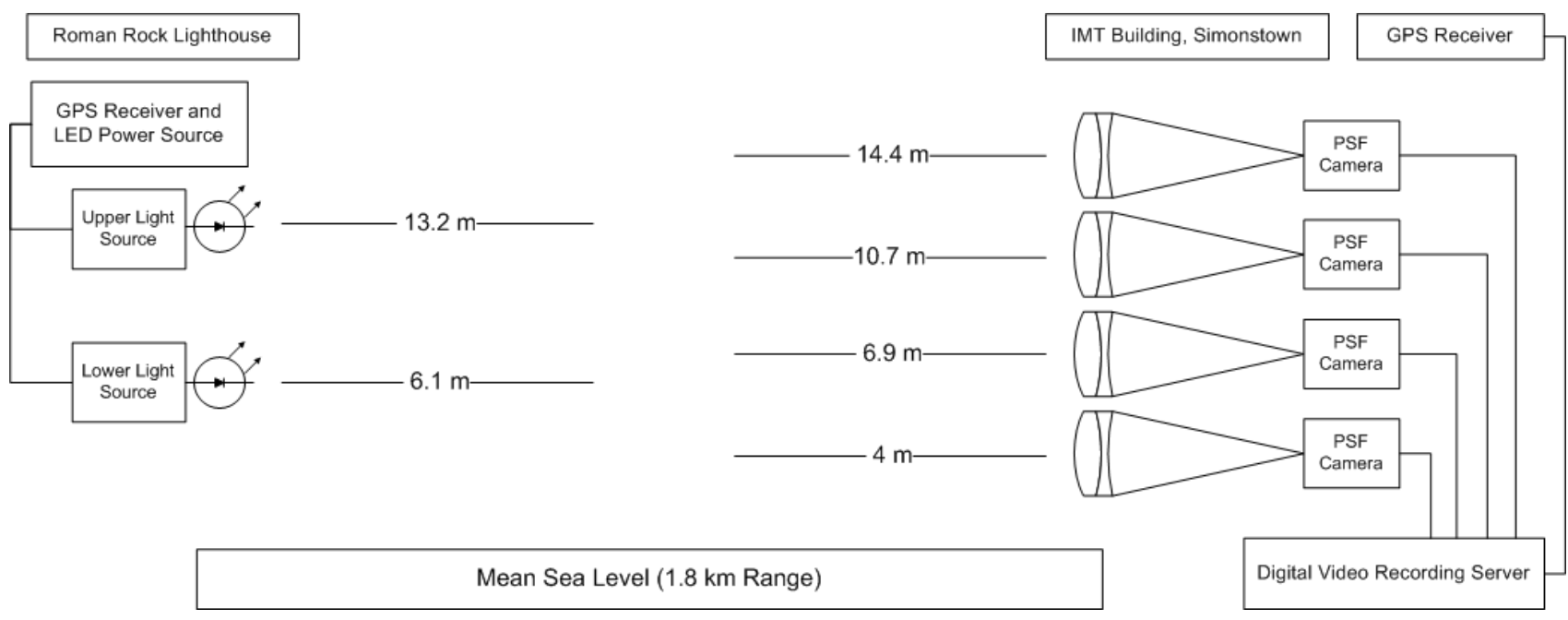

Figure 1: Atmospheric MTF Experimental Layout

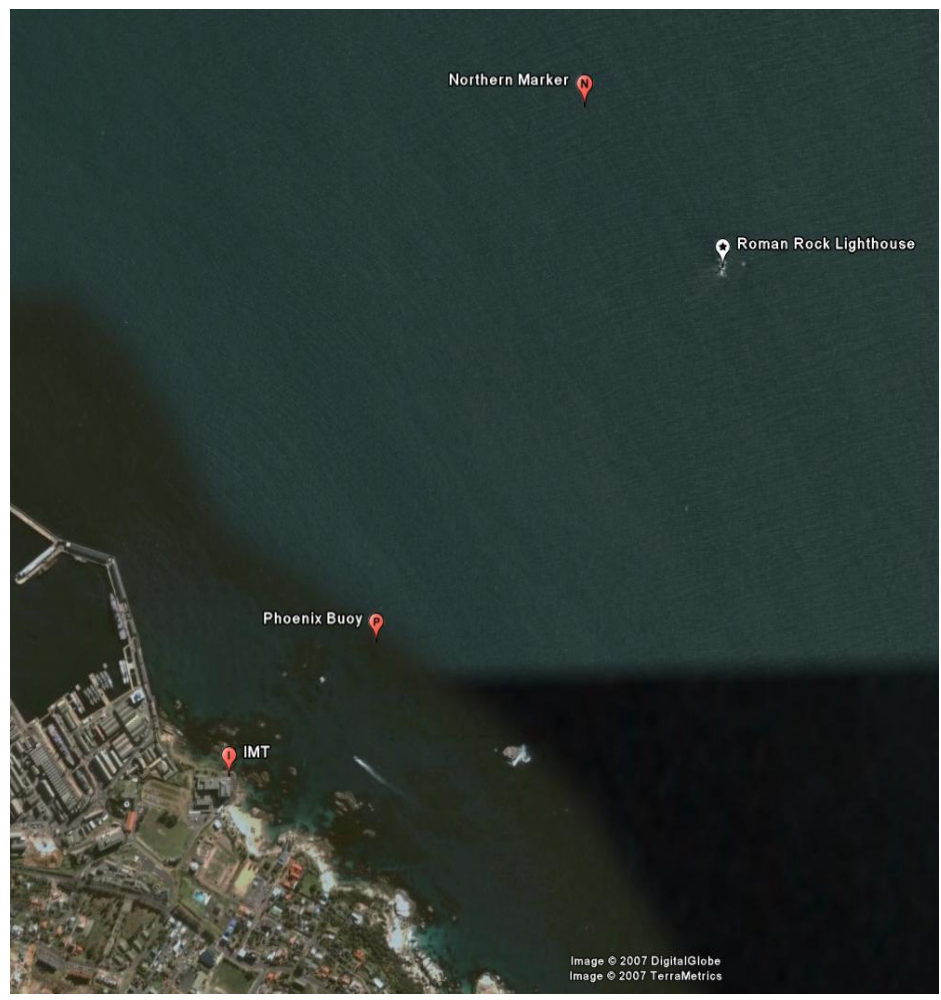

Figure 2: Simonstown, IMT and Roman Rock, Courtesy Google Earth

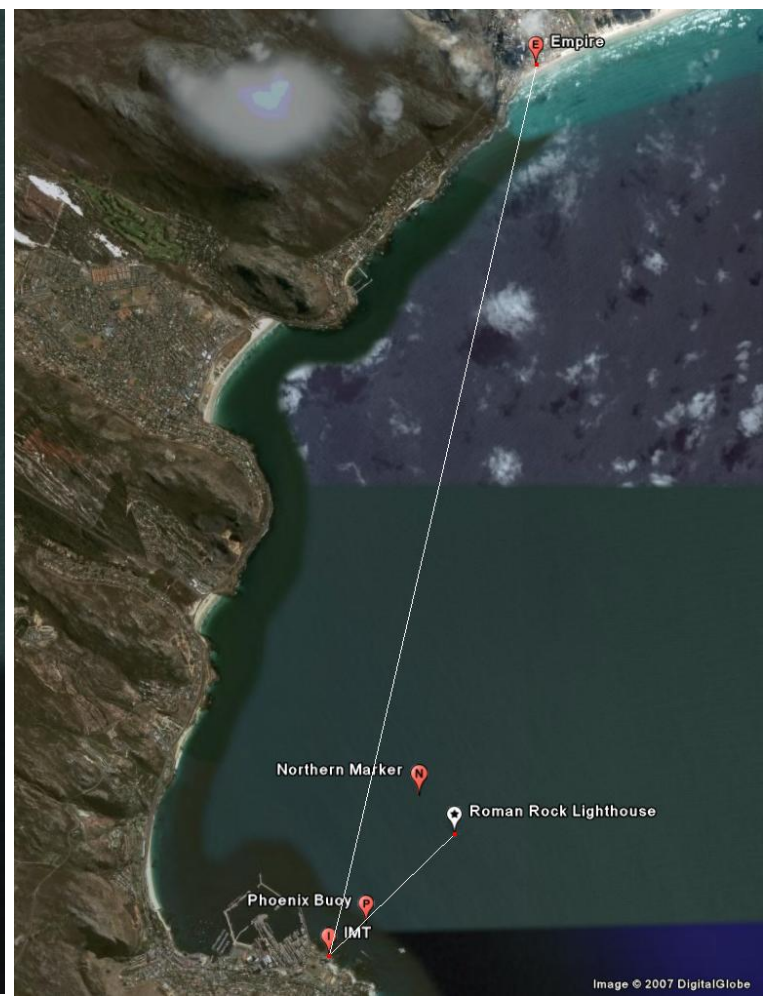

Figure 3: Simonstown, Muizenberg and Roman Rock, Courtesy Google Earth 


\section{EXPERIMENTAL HARDWARE DESCRIPTION}

The light sources on Roman Rock comprised high intensity light emitting diodes (LEDs, in this case the Lumileds Luxeon III lambertian emitters) operating at a central wavelength of $630 \mathrm{~nm}$ and full width at half maximum (FWHM) of $36 \mathrm{~nm}$. These sources were chosen for high efficiency and compactness in the face of the formidable constraints of working on Roman Rock. The LEDs were equipped with protective baffles, heat sinks and plastic collimator lenses to increase intensity at the receiver telescopes. The collimator aperture was $22 \mathrm{~mm}$ in diameter with an FWHM beam divergence of $8^{\circ}$ and an axial photometric amplification of $16.5 \mathrm{~cd} / \mathrm{lm}$. The LED in question has a specified photometric flux output of $190 \mathrm{~lm}$ (minimum of $120 \mathrm{~lm}$ ). The axial intensity of the source was therefore predicted at about $3130 \mathrm{~cd}$ nominal. This could not be verified directly, but the typical exposure time for the camera was close to what was predicted. The LEDs were driven by a current controller from a battery power supply. The light sources were switched on for 10 seconds every minute by a microcontroller, during which recording of images took place. Synchronization was achieved through the use of Global Positioning System (GPS) receivers both at the sources and at the telescopes at IMT.

The telescopes were of identical design and comprised an achromatic doublet of aperture $46.5 \mathrm{~mm}$ diameter and nominal focal length of $1000 \mathrm{~mm}$. The focal ratio was chosen so as to place the optical cut-off frequency within the Nyquist frequency for the CCD pixel pitch. The doublet, together with a bandpass filter and the camera were mounted in a mild steel tube, chosen for relatively low thermal expansion. The steel tube was suspended, but not mechanically constrained in a thick-wall, white plastic tube with a large air-gap between the steel and plastic tubes. This was done to help stabilize and homogenize the thermal environment of the steel tube especially while the telescope was subject to direct sunlight.

The four telescopes were suspended from the ceilings of the balcony parapets of the IMT building as shown in Figure 4 and Figure 5.

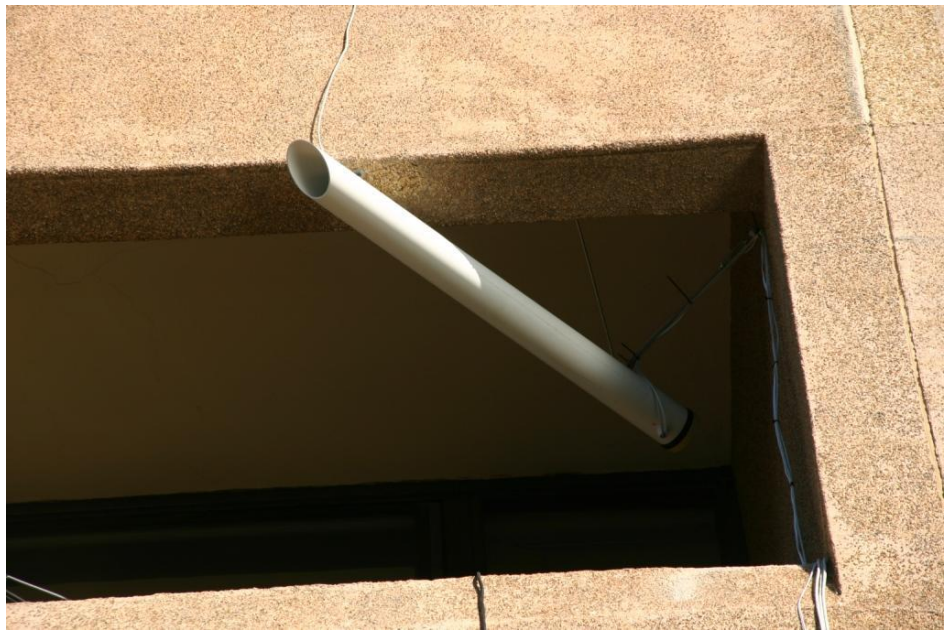

Figure 4: Receiver Telescope Mounted at IMT

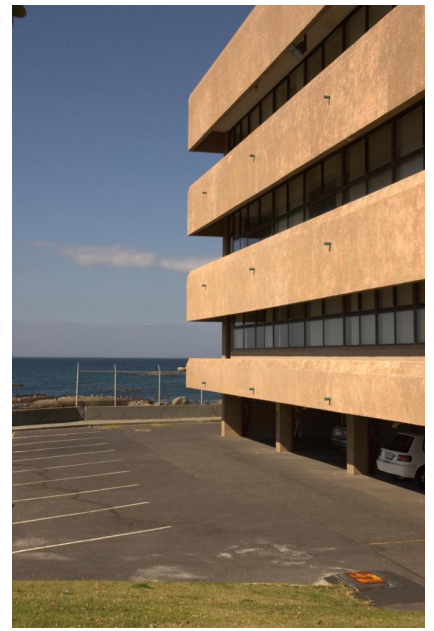

Figure 5: Balcony Parapets at IMT

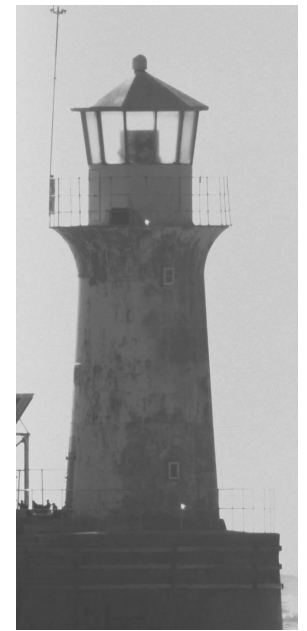

Figure 6: Image of Roman Rock

The bandpass filters mounted in the telescopes in front of the cameras had a central wavelength of $633 \mathrm{~nm}$ and an FWHM of $40 \mathrm{~nm}$. This was matched to the emission spectrum of the sources in order to reduce background radiation.

The optical quality of the achromats and filters were determined through MTF and wavefront distortion measurements using interferometry at $633 \mathrm{~nm}$.

The cameras were the Prosilica model GC1380M, which incorporates the Sony ICX285AL CCD. The cameras were verified for linearity and spatial uniformity of photoresponse using an integrating sphere. Camera MTF was measured at 
central wavelengths of $600 \mathrm{~nm}$ and $800 \mathrm{~nm}$ by the slanted slit projection technique. The camera MTF at both these wavelengths conformed very closely to a sinc function corresponding to the CCD pixel pitch of $6.45 \mu \mathrm{m}$ (horizontal and vertical) and $100 \%$ fill factor. These cameras can produce digital monochrome images of 14 bit resolution via a gigabit Ethernet interface. The data feeds from all four cameras were cabled to a single digital image data acquisition server. A utility was written using the Prosilica software development kit to acquire the images.

\section{DATA ACQUISITION}

Data was acquired every minute from noon on Tuesday 5 June 2007 until shortly after sunrise on Wednesday 13 June 2007. A wide range of meteorological conditions ${ }^{1}$ were encountered during this period. The LEDs were switched on for a period of 10 seconds at the start of the GPS minute. While the sources were switched on, ten images from each telescope were recorded at a typical framerate of $15 \mathrm{~Hz}$. Both sources fell within the fields of view of all telescopes. Once the sources had been turned off, another ten background images were recorded from each telescope. To reduce disk space requirements, a sub-frame around each source was cut out and stored. Both source-on and background images subframes were recorded. An exposure time of $1.5 \mathrm{~ms}$ on the cameras was used throughout, which would generally correspond to the short exposure regime. A longer exposure image with the LED sources switched on is shown above in Figure 6. The sources, which can be seen on the upper and lower balconies, are saturated in this image.

Data quality was compromised on a few occasions. Signal flux was very low during the heavier rain showers. On imagery from some of the telescopes, the lighthouse lamp had to be masked out on the images acquired at night. A large boat obscured the sources on one occasion. On clear mornings there was evidence that dynamic solar reflections (glint/glitter) off the ocean had an adverse effect when the sun was positioned near the telescope sightlines. On 12 June the flux from the lower source suddenly dropped at around 11:00 am. On retrieval from Roman Rock, the lower source was found to be out of alignment. We speculate that a seabird had alighted on the source baffle on 12 June.

\section{DATA PROCESSING}

Image data was post-processed using Matlab. Several metrics were extracted, most notably an MTF Strehl ratio as a measure of image quality and then also a scintillation index. The fluctuation in the centroid position of the PSF was also evaluated and this is related to wavefront angle of arrival (AoA) fluctuations in a linear manner.

The Strehl ratio is popular amongst astronomers as a figure of merit for adaptive optics systems. Theoretically, the Strehl ratio is well-defined but there are important issues with regard to practical computation (Roberts et.al. ${ }^{2}$ ). In this case the MTF Strehl ratio will be understood to mean the ratio of the measured volume under the normalized MTF to the maximum volume based on the system MTF only. While Roberts et. el. discourage the use of the MTF Strehl ratio in favour of the OTF Strehl ratio we justify use on the basis that we are only trying to establish image quality trends within our own measurements as opposed to performing inter-comparisons with other workers. Hence, we used the working definition given in Equation (1).

$$
S=\frac{\iint M_{\text {meas }}\left(f_{x}, f_{y}\right) d f_{x} d f_{y}}{\iint M_{\text {perf }}\left(f_{x}, f_{y}\right) d f_{x} d f_{y}}
$$

Where $S$ is the MTF Strehl ratio, $M_{\text {perf }}$ is the system MTF without atmospheric influences, $M_{\text {meas }}$ is the measured MTF and $\left(f_{x}, f_{y}\right)$ are the coordinates in spatial frequency space. $M_{\text {perf }}$ is plotted as the "System MTF" in Figure 7, and is computed as the product of the source spatial spectrum, the lens MTF and the CCD MTF. 


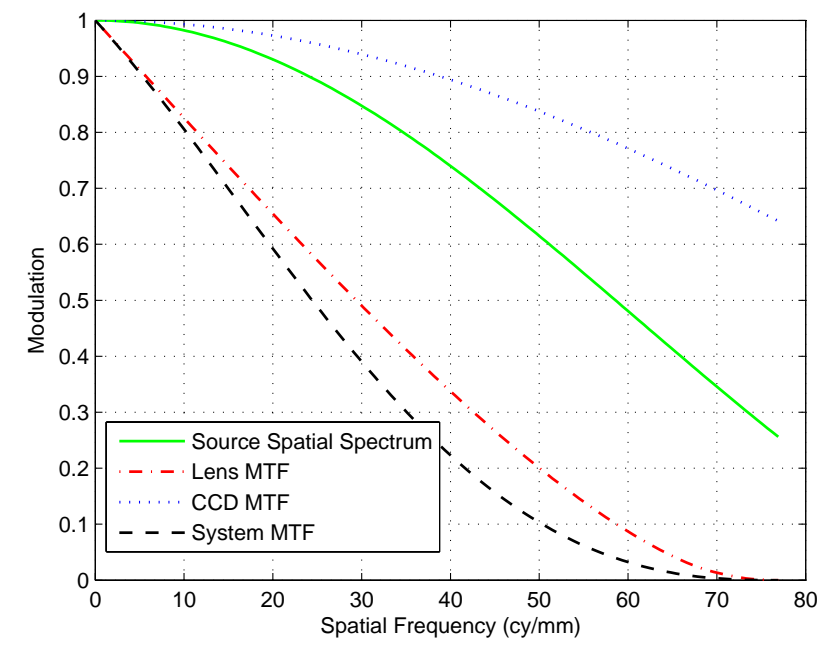

Figure 7: System MTF Expressed at the CCD

Under very good atmospheric conditions, MTF Strehl ratios as computed above sometimes exceeded unity in our dataset. There are several possible explanations for this. For one, the spatial modulation of the source could be higher than that shown in Figure 7, due to internal structure. The curve in Figure 7 should be considered a minimum (for a uniformly illuminated source of $22 \mathrm{~mm}$ diameter). Also, there could conceivably be super-resolution effects induced by local atmospheric refraction due to small bubbles in the line of sight.

The scintillation index, $\beta$, was estimated from the imagery by computing the total energy signal in the PSF (after background subtraction) for each of the ten images captured per minute, per telescope and per source and taking the ratio of the variance to the square of the mean signal i.e. $\beta=\sigma_{I}^{2} /\langle I\rangle^{2}$. No aperture or time averaging effects were taken into account.

\section{DISCUSSION AND RESULTS}

Our expectation was that Strehl ratios would increase (meaning improved image quality) and the scintillation index would decrease with increasing effective atmospheric path height above the sea surface. For the complete dataset, both MTF Strehl ratio and scintillation index showed clear height trends, but the Strehl trend was inverted to what was expected i.e. image quality deteriorated with height. The average trends for MTF Strehl ratio and scintillation with respect to effective atmospheric path height are shown in Figure 8. Note that the scale is correct for the Strehl ratio in Figure 8 , but the scintillation index is relative. Scintillation indices in this experiment were typically less than 0.08 . The reason for this inverted trend in MTF Strehl ratio is thought to be related to the range weighting factors for scintillation versus MTF and this aspect will be explored next.

Amplitude variance, $\sigma_{x}^{2}$, for a spherical electro-magnetic wave propagating in a weakly turbulent atmosphere can be computed from the atmospheric refractive index structure function parameter $C_{n}^{2}$ as follows (Kopeika ${ }^{3}$, Chapter 15).

$$
\sigma_{x}^{2}=0.56 k^{7 / 6} \int_{0}^{L} C_{n}^{2}(z)\left(\frac{z}{L}\right)^{5 / 6}(L-z)^{5 / 6} d z
$$

Where $k$ is the wavenumber, $z$ is the distance from the source to the receiver and $L$ is the total atmospheric path length. The weighting factor of $C_{n}^{2}$ inside the integral in Equation (2) is plotted in Figure 9. This range weighting factor for amplitude variance is symmetrical with respect to source and receiver and peaks in the centre of the atmospheric 
path. The scintillation index will therefore also have a symmetrical range weighting factor, peaking at the centre of the path. MTF, however is more sensitive to wavefront deformations introduced near the receiver telescope. This is evident from the long exposure atmospheric turbulence MTF, $M_{T L}$, which depends on $C_{n}^{2}$ as follows.

$$
M_{T L}\left(f_{a}\right)=\exp \left[-57.53 f_{a}^{5 / 3} \lambda^{-1 / 3} \int_{0}^{L} C_{n}^{2}(z)\left(\frac{z}{L}\right)^{5 / 3} d z\right]
$$

Where $f_{a}$ is (angular) spatial frequency and $\lambda$ is wavelength. The range weighting factor inside the integral in Equation (3) is also plotted in Figure 9. The variance of the AoA, $\left\langle\alpha^{2}\right\rangle$, has the same range weighting as the MTF as seen in the following relation.

$$
\left\langle\alpha^{2}\right\rangle=2.914 D^{-1 / 3} \int_{0}^{L} C_{n}^{2}(z)\left(\frac{z}{L}\right)^{5 / 3} d z
$$

Where $D$ is the receiver optics aperture diameter.

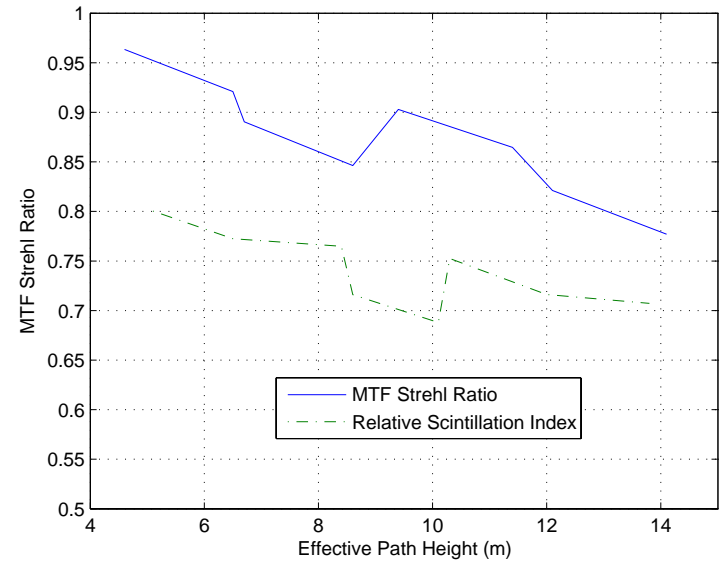

Figure 8: Trends in MTF and Scintillation with Path Height

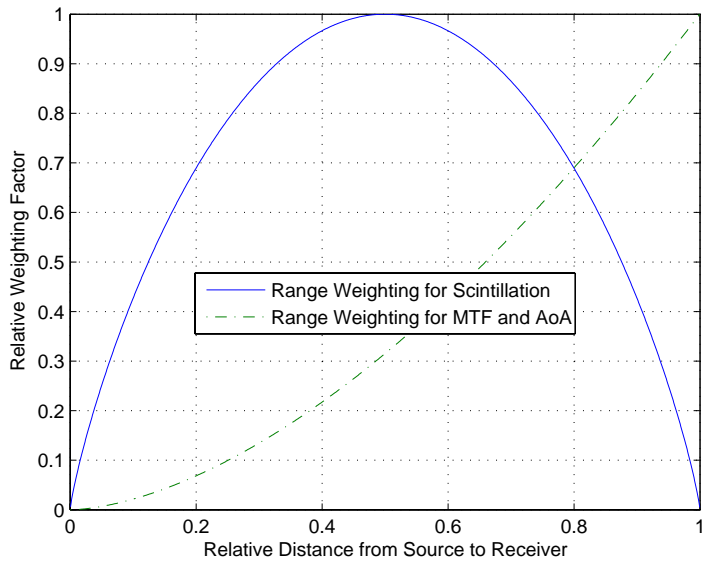

Figure 9: Range Weighting for Scintillation and MTF/AoA

Since the range weighting factor is symmetrical for scintillation, the effective atmospheric path height is just the mean of the starting and ending heights. For MTF Strehl and variance of AoA, the effective path height is computed as a weighted mean. The mean and weighted path heights are given below in Table 1 for each of the 8 paths from the sources to the receivers. The effective path height order is seen to be different for scintillation and MTF/AoA.

Figure 8 also suggests that an effective path height of between $9 \mathrm{~m}$ and $10 \mathrm{~m}$ would be a good choice for short/medium range surveillance, but only in the particular scenario of this experiment. A general conclusion in respect of optimal path height cannot be reached using this dataset.

Table 1: Effective Atmospheric Path Heights for Scintillation and MTF/AoA

\begin{tabular}{|l|c|c|c|c|}
\hline & $\begin{array}{c}\text { Lower Source (6.1 m) } \\
\text { Weighted for MTF/AoA (m) }\end{array}$ & $\begin{array}{c}\text { Lower Source (6.1 m) } \\
\text { Unweighted, Scintillation (m) }\end{array}$ & $\begin{array}{c}\text { Upper Source (13.2 m) } \\
\text { Weighted for MTF/AoA (m) }\end{array}$ & $\begin{array}{c}\text { Upper Source (13.2 m) } \\
\text { Unweighted, Scintillation (m) }\end{array}$ \\
\hline Telescope 1 (4 m) & 4.6 & 5.1 & 6.5 & 8.6 \\
\hline Telescope 2 (6.9 m) & 6.7 & 6.5 & 8.6 & 10.1 \\
\hline Telescope 3 (10.7 m) & 9.4 & 8.4 & 11.4 & 12.0 \\
\hline Telescope 4 (14.4 m) & 12.1 & 10.3 & 14.1 & 13.8 \\
\hline
\end{tabular}

Differences in temporal and spatial trends between scintillation index and image quality could be attributed to several causes. Firstly, aerosols directly affect overall MTF (Kopeika ${ }^{3}$, Chapter 17) but have an indirect effect on scintillation, 
mediated through increased or decreased bulk atmospheric radiative heat loading. Secondly, and this is thought to be a major factor in this experiment, different distributions of turbulence strength along the atmospheric path can cause divergent trends in scintillation versus image quality because of the different range weighting factors as outlined above. AoA fluctuations are driven largely by turbulence as for scintillation, but have the same range weighting factor as MTF. By evaluating all three (scintillation, AoA and Strehl ratio), it is possible to start discriminating the effects of aerosols from the effects of turbulence on image quality. Some results for 11 June 2007 are presented in Figure 10 and Figure 11.

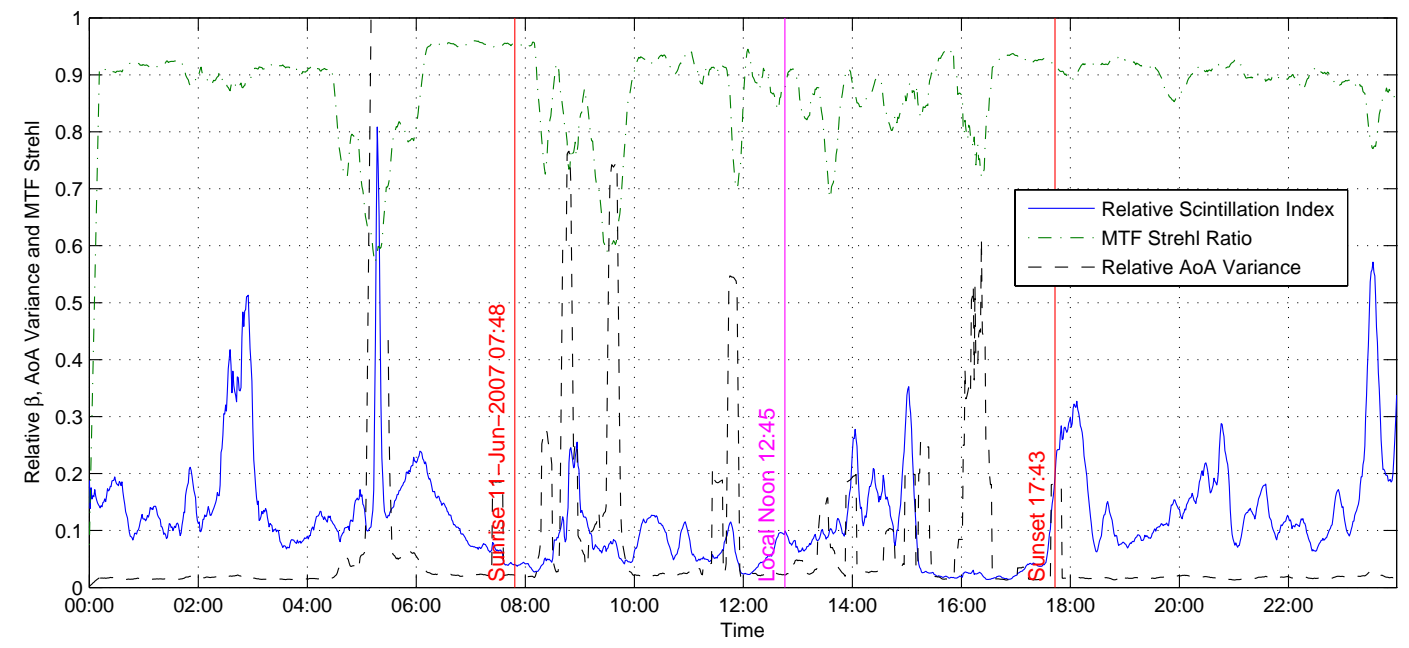

Figure 10: Relative Scintillation, MTF Strehl and Relative AoA Variance for 11 June 2007

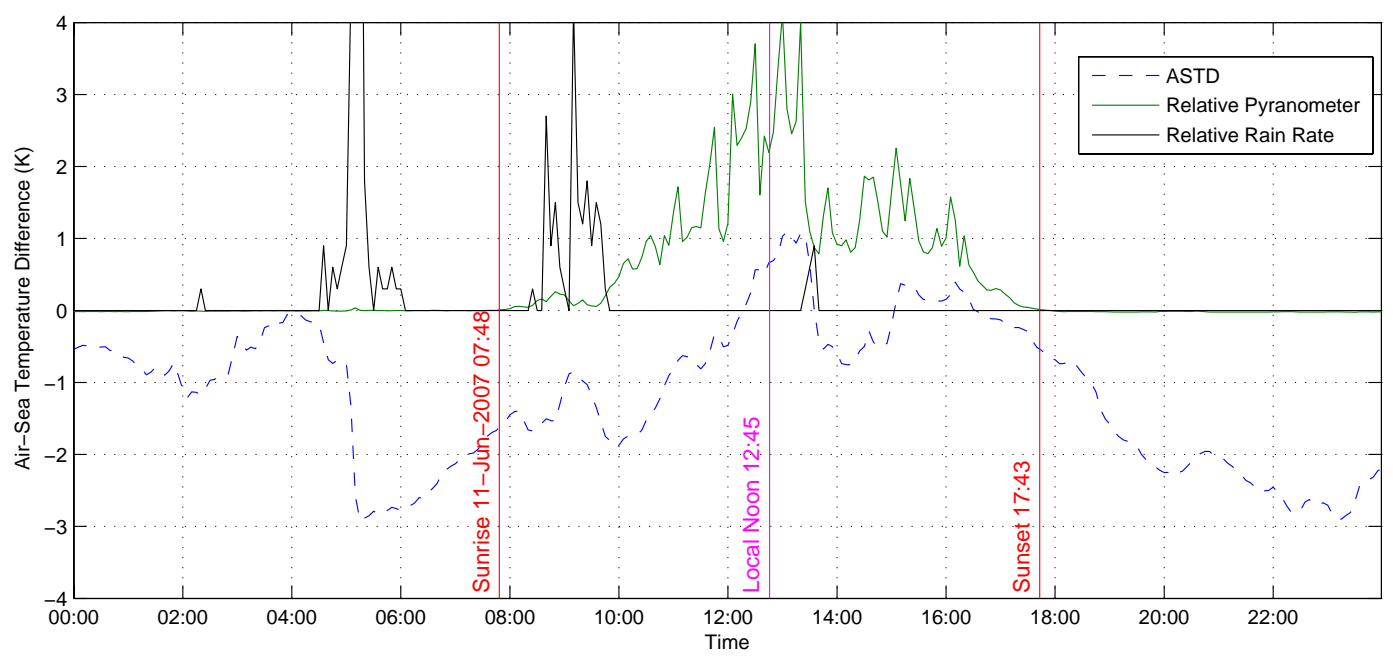

Figure 11: Air-Sea Temperature Difference, Relative Pyranometer and Relative Rain Rate for 11 June 2007

Note again that only the Strehl ratio in Figure 10 and the air-sea temperature difference (ASTD) in Figure 11 are plotted to absolute scale. All other curves are plotted in a relative sense to illustrate the temporal variations.

On 11 June 2007 there were two major and two minor rain showers as shown on Figure 11. Image quality as measured by MTF Strehl was strongly affected during rain, and variance of AoA was generally correlated much more closely with image quality than with scintillation. However, the sudden large increase in AoA during the peak of the early morning shower is thought to be due to poor signal and the general loss in image quality due to aerosol (rain) scattering. The AoA events just before noon and in the late afternoon are clearly related to turbulence. Scintillation generally peaked after rain events and the scintillation spike during the early morning shower is also associated with very poor signal. 
Of particular interest in Figure 10 is the degradation of image quality between 16:00 and 17:00, again correlated with strong variance of AoA, but little scintillation. This is very likely caused by turbulence occurring near the telescope apertures. Solar exposure of the northern wall of the IMT building (Figure 5) where the telescopes were located is suspected to be the cause. Total downwelling shortwave radiation was obtained from a pyranometer belonging to TNO. Note that the pyranometer gives the irradiance on a horizontal surface. A vertical north-facing wall at this time of day would have much higher irradiance.

\section{CONCLUSIONS}

From our work we derive the following conclusions:

a) Under otherwise good atmospheric conditions, image quality in this experiment is thought to be strongly driven by turbulence occurring near the telescopes. The inverted height dependence of MTF Strehl ratio (deteriorating image quality with height) is believed to be caused by a sheet of relatively warm, turbulent air rising up the north wall of the IMT building, caused by shortwave irradiance. As the volume of air rises, the warm wall contributes progressively more heat and more turbulence.

b) This finding adds weight to the importance of carefully evaluating the local thermal and turbulence environment of imaging systems for surveillance. Scintillometry is not the appropriate tool for this evaluation.

c) For imaging applications, scintillation, AoA and Strehl ratio (and other image quality metrics) should all be evaluated in order to have a more complete picture of what is happening in the atmospheric path.

The following improvements to this type of experiment would in future be considered:

a) Denser sampling of the PSF (camera with smaller pixels or lower telescope cut-off frequency). This should produce more reliable extraction of the Strehl ratios (Roberts et. al. ${ }^{2}$ ) for purposes of inter-comparison.

b) Adaptation of camera exposure time during periods of reduced visibility and implementation of brighter sources in order to stay in the short exposure regime.

c) Avoid observation sightlines that allow periods during which straylight from the solar glitter path can enter the telescopes. Alternatively, improve the straylight characteristics of the telescopes using more judicious baffling.

d) Perform more data processing and "quick-look" displays during acquisition for improved experimental monitoring.

e) Better characterize the spatial spectrum of the source.

f) Take control of the local thermal environment of the receiver telescopes.

\section{ACKNOWLEDGEMENTS}

The work for this paper was supported by Armscor, South Africa. Dirk Bezuidenhout motivated the experiment and provided support in various ways. Danie van Staden assisted with construction and characterization of the experimental hardware. Software was written by Bernhardt Duvenhage and Ludi Kruger. We acknowledge the Institute for Maritime Technology, especially Willie Gunter and the South African Navy for their assistance. We thank the South African Port Authority for giving us access to Roman Rock Lighthouse.

\section{REFERENCES}

[1] Schwering, P.B.W, Bezuidenhout, D.F., Gunter, W.H., de Jong, A.N., Fritz, P.J., le Roux, F.P.J, Sieberhagen, R.H., Holloway, M, Vrahimis, G., October, F.J, Kemp, R.A.W., "Optical Characterisation of small surface targets", Proc. SPIE 6739, 67390H (2007).

[2] Roberts, L.C. Jr., Perrin, M.D., Marchis,F., Sivaramakrishnan, A., Makidon, R.B., Christou, J.C., Macintosh, B.C., Poyneer, L.A., van Dam, L.A., and Troy, M., "Is That Really Your Strehl Ratio?”, Proc. SPIE 5490, 504 (2004).

[3] Kopeika, N.S., [A System Engineering Approach to Imaging], SPIE Press, Bellingham, Washington (1998). 